\title{
Status of the aligned two-Higgs-doublet model confronted with the Higgs data
}

\author{
Lei Wang and Xiao-Fang Han \\ Department of Physics, Yantai University, \\ Yantai 264005, PR China \\ E-mail: leiwang@ytu.edu.cn, xfhan@mail.itp.ac.cn
}

ABSTRACT: Imposing the theoretical constraints from vacuum stability, unitarity and perturbativity as well as the experimental constraints from the electroweak precision data, flavor observables and the non-observation of additional Higgs at collider, we study the implications of available Higgs signals on a two-Higgs-doublet model with the alignment of the down-type quarks and charged lepton Yukawa coupling matrices. Compared to the four traditional types of two-Higgs-doublet models, the model has two additional mixing angles $\theta_{d}$ and $\theta_{l}$ in the down-type quark and charged lepton Yukawa interactions. We find that the mixing angle $\theta_{d}$ can loose the constraints on $\sin (\beta-\alpha), \tan \beta$ and $m_{H^{ \pm}}$sizably. The model can provide the marginally better fit to available Higgs signals data than SM, which requires the Higgs couplings with gauge bosons, $u \bar{u}$ and $d \bar{d}$ to be properly suppressed, and favors $\left(1<\theta_{d}<2,0.5<\theta_{l}<2.2\right)$ for $m_{h}=125.5 \mathrm{GeV}$ and $\left(0.5<\theta_{d}<2,0.5<\theta_{l}<2.2\right)$ for $m_{H}=125.5 \mathrm{GeV}$. However, these Higgs couplings are allowed to have sizable deviations from $\mathrm{SM}$ for $\left(m_{h}=125.5 \mathrm{GeV}, 125.5 \leq m_{H} \leq 128 \mathrm{GeV}\right)$ and $\left(125 \mathrm{GeV} \leq m_{h} \leq 125.5 \mathrm{GeV}\right.$, $\left.m_{H}=125.5 \mathrm{GeV}\right)$.

Keywords: Higgs Physics, Beyond Standard Model

ARXIV EPRINT: 1312.4759 


\section{Contents}

1 Introduction 1

2 Aligned two-Higgs-doublet model 2

3 Numerical calculations 3

4 Results and discussions $\quad 4$

4.1 Scenario A 4

4.2 Scenario B 8

5 Conclusion $\quad 13$

\section{Introduction}

The CMS and ATLAS collaborations have announced the observation of a scalar around $125 \mathrm{GeV}[1,2]$, which is supported by the Tevatron search [3]. The properties of this particle with large experimental uncertainties are well consistent with the SM Higgs boson, which will give the strong constraints on the effects of new physics.

One of the simplest extension of the SM is obtained by adding a second $\mathrm{SU}(2)_{L}$ Higgs doublet [4]. The two-Higgs-doublet model (2HDM) has very rich Higgs phenomenology, including two neutral CP-even Higgs bosons $h$ and $H$, one neutral pseudoscalar $A$, and two charged Higgs $H^{ \pm}$. Further, the couplings of the CP-even Higgs bosons can deviate from SM Higgs boson sizably. Therefore, the observed signal strengths of the Higgs boson and the non-observation of additional Higgs can give the strong implications on the 2HDMs. The 2HDMs generically have tree-level flavor changing neutral currents (FCNC), which can be forbidden by a discrete symmetry. There are four types for $2 \mathrm{HDMs}$, which are typically called the Type-I [5, 6], Type-II [5, 7], Lepton-specific, and Flipped models [8-13] according to their different Yukawa couplings. In light of the recent Higgs data, there have been various studies on these 2HDMs over the last few months [14-26].

In this paper, we focus on a two-Higgs-doublet model that allows both doublets to couple to the down-type quarks and charged leptons with aligned Yukawa matrices ( A2HDM) [23, 27]. Also there is no tree-level FCNC in this model. Compared to the above four types of $2 \mathrm{HDMs}$, there are two additional mixing angles in the Yukawa couplings of the down-type quarks and charged leptons. This model can be mapped to the four types of $2 \mathrm{HDMs}$ for the two angles are taken as specific values. There are also some works on the Higgs properties in the A2HDM after the discovery of Higgs boson [23, 24, 28-32]. After imposing the theoretical constraints from vacuum stability, unitarity and perturbativity as well as the experimental constraints from the electroweak precision data, flavor observables 
and the non-observation of additional Higgs at collider, we study the implication of the latest Higgs signals data on the A2HDM.

Our work is organized as follows. In section 2 we recapitulate the A2HDM. In section 3 we introduce the numerical calculations. In section 4 , we discuss the implications of the available Higgs signals on the A2HDM after imposing the theoretical and experimental constraints. Finally, we give our conclusion in section V.

\section{Aligned two-Higgs-doublet model}

The general Higgs potential is written as [33]

$$
\begin{aligned}
\mathrm{V}= & m_{11}^{2}\left(\Phi_{1}^{\dagger} \Phi_{1}\right)+m_{22}^{2}\left(\Phi_{2}^{\dagger} \Phi_{2}\right)-\left[m_{12}^{2}\left(\Phi_{1}^{\dagger} \Phi_{2}+\text { h.c. }\right)\right] \\
& +\frac{\lambda_{1}}{2}\left(\Phi_{1}^{\dagger} \Phi_{1}\right)^{2}+\frac{\lambda_{2}}{2}\left(\Phi_{2}^{\dagger} \Phi_{2}\right)^{2}+\lambda_{3}\left(\Phi_{1}^{\dagger} \Phi_{1}\right)\left(\Phi_{2}^{\dagger} \Phi_{2}\right)+\lambda_{4}\left(\Phi_{1}^{\dagger} \Phi_{2}\right)\left(\Phi_{2}^{\dagger} \Phi_{1}\right) \\
& +\left[\frac{\lambda_{5}}{2}\left(\Phi_{1}^{\dagger} \Phi_{2}\right)^{2}+\text { h.c. }\right]+\left[\lambda_{6}\left(\Phi_{1}^{\dagger} \Phi_{1}\right)\left(\Phi_{1}^{\dagger} \Phi_{2}\right)+\text { h.c. }\right] \\
& +\left[\lambda_{7}\left(\Phi_{2}^{\dagger} \Phi_{2}\right)\left(\Phi_{1}^{\dagger} \Phi_{2}\right)+\text { h.c. }\right]
\end{aligned}
$$

We focus on the CP-conserving model in which all $\lambda_{i}$ and $m_{12}^{2}$ are real. Further, we assume $\lambda_{6}=\lambda_{7}=0$, which also facilitates the comparison to the four traditional types of 2HDMs. The two complex scalar doublets have the hypercharge $Y=1$,

$$
\Phi_{1}=\left(\begin{array}{c}
\phi_{1}^{+} \\
\frac{1}{\sqrt{2}}\left(v_{1}+\phi_{1}^{0}+i a_{1}\right)
\end{array}\right), \quad \Phi_{2}=\left(\begin{array}{c}
\phi_{2}^{+} \\
\frac{1}{\sqrt{2}}\left(v_{2}+\phi_{2}^{0}+i a_{2}\right)
\end{array}\right) .
$$

Where $v_{1}$ and $v_{2}$ are the electroweak vacuum expectation values (VEVs) with $v^{2}=v_{1}^{2}+v_{2}^{2}=$ $(246 \mathrm{GeV})^{2}$. The ratio of the two VEVs is defined as usual to be $\tan \beta=v_{2} / v_{1}$. After spontaneous electroweak symmetry breaking, the physical scalars are two neutral CP-even $h$ and $H$, one neutral pseudoscalar $A$, and two charged scalar $H^{ \pm}$. These scalars are also predicted in the Higgs triplet models [34-36].

The Yukawa interactions of the Higgs doublets with the SM fermions can be given by

$$
\begin{aligned}
-\mathcal{L}= & y_{u} \bar{Q}_{L} \tilde{\Phi}_{2} u_{R}+y_{d} \bar{Q}_{L}\left(\cos \theta_{d} \Phi_{1}+\sin \theta_{d} \Phi_{2}\right) d_{R} \\
& +y_{l} \bar{l}_{L}\left(\cos \theta_{l} \Phi_{1}+\sin \theta_{l} \Phi_{2}\right) e_{R}+\text { h.c. }
\end{aligned}
$$

where $Q^{T}=\left(u_{L}, d_{L}\right), L^{T}=\left(\nu_{L}, l_{L}\right)$, and $\widetilde{\Phi}_{2}=i \tau_{2} \Phi_{2}^{*} . y_{u}, y_{d}$ and $y_{\ell}$ are $3 \times 3$ matrices in family space. $\theta_{d}$ and $\theta_{l}$ parameterize the two Higgs doublets couplings to down-type quarks and charged leptons, respectively. Where a freedom is used to redefine the two linear combinations of $\Phi_{1}$ and $\Phi_{2}$ to eliminate the coupling of the up-type quarks to $\Phi_{1}$ [23].

The tree-level couplings of the neutral Higgs bosons can have sizable deviations from those of SM Higgs boson. Table 1 shows the couplings of neutral Higgs bosons with respect to the SM Higgs boson. According to table 1, the A2HDM can be mapped to the four traditional types of $2 \mathrm{HDMs}$ via the angles $\theta_{d}$ and $\theta_{l}$ specified in table 2. 


\begin{tabular}{|c|c|c|c|c|}
\hline & $V V(W W, Z Z)$ & $u \bar{u}$ & $d \bar{d}$ & $l \bar{l}$ \\
\hline$h$ & $\sin (\beta-\alpha)$ & $\frac{\cos \alpha}{\sin \beta}$ & $-\frac{\sin \left(\alpha-\theta_{d}\right)}{\cos \left(\beta-\theta_{d}\right)}$ & $-\frac{\sin \left(\alpha-\theta_{l}\right)}{\cos \left(\beta-\theta_{l}\right)}$ \\
$H$ & $\cos (\beta-\alpha)$ & $\frac{\sin \alpha}{\sin \beta}$ & $\frac{\cos \left(\alpha-\theta_{d}\right)}{\cos \left(\beta-\theta_{d}\right)}$ & $\frac{\cos \left(\alpha-\theta_{l}\right)}{\cos \left(\beta-\theta_{l}\right)}$ \\
$A$ & 0 & $-\frac{i}{\tan \beta} \gamma_{5}$ & $-i \tan \left(\beta-\theta_{d}\right) \gamma_{5}$ & $-i \tan \left(\beta-\theta_{l}\right) \gamma_{5}$ \\
\hline
\end{tabular}

Table 1. The tree-level couplings of the neutral Higgs bosons with respect to those of the SM Higgs boson. $u, d$ and $l$ denote the up-type quarks, down-type quarks and the charged leptons, respectively. The angle $\alpha$ parameterizes the mixing of two CP-even Higgses $h$ and $H$.

\begin{tabular}{|c|c|c|c|c|}
\hline & Type I & Type II & Lepton-specific & Flipped \\
\hline$\theta_{d}$ & $\frac{\pi}{2}$ & 0 & $\frac{\pi}{2}$ & 0 \\
$\theta_{l}$ & $\frac{\pi}{2}$ & 0 & 0 & $\frac{\pi}{2}$ \\
\hline
\end{tabular}

Table 2. The values of mixing angles $\theta_{d}$ and $\theta_{l}$ for the four traditional types of $2 \mathrm{HDMs}$.

\section{Numerical calculations}

We have employed the following four codes to implement the various theoretical and experimental constraints. We require the A2HDM to explain the experimental data of flavor observables and the electroweak precision data within $2 \sigma$ range.

- 2HDMC-1.5 [37, 38]: the code is used to implement the theoretical constraints from the vacuum stability, unitarity and coupling-constant perturbativity. Also the oblique parameters $(S, T, U)$ and $\delta \rho$ are calculated and the corresponding experimental data are from [39]. $\delta \rho$ has been measured very precisely via Z-pole precision observables to be very close to 1 , which imposes a strong constraint on the mass difference between the various Higgses in $2 \mathrm{HDM}$. In addition, the code $2 \mathrm{HDMC}-1.5,{ }^{1}$ which calculates the Higgs couplings and the decay branching fractions, provides the necessary inputs for the following three codes.

- Superlso-3.3 [40]: the code is used to implement the constraints from flavor observables, including $B \rightarrow X_{s} \gamma$ [41], $B_{s} \rightarrow \mu^{+} \mu^{-}$[42], $B_{u} \rightarrow \tau \nu$ [43] and $D_{s} \rightarrow \tau \nu$ [41]. Also the constrains from $\Delta m_{B_{d}}$ and $\Delta m_{B_{s}}$ are considered, ${ }^{2}$ which are calculated using the formulas in [44].

- HiggsBounds-4.1.0 [45, 46]: the code is used to implement the exclusion constraints from the neutral and charged Higgses searches at LEP, Tevatron and LHC at 95\% confidence level.

- HiggsSignals-1.1.0 [47, 48]: the code is used to perform a global $\chi^{2}$ fit to the most up-to-date signal strength measurements as of November 2013. We consider the

\footnotetext{
${ }^{1} \mathrm{~A}$ bug is modified: $\Gamma(h \rightarrow Z \gamma)=0$ for $m_{h}<m_{Z}$.

${ }^{2}$ Particle Data Group, 2013 partial update for the 2014 edition.
} 
73 Higgs signal strengths observables from ATLAS [49-57], CMS [58-70], CDF [71] and D0 [72] collaborations as well as the four Higgs mass measurements from the ATLAS and CMS $h \rightarrow \gamma \gamma$ and $h \rightarrow Z Z^{*} \rightarrow 4 l$ analyses, which are listed in the [48]. In our discussions, we will pay particular attention to the surviving samples with $\chi^{2}-\chi_{\min }^{2} \leq 6.18$, where $\chi_{\min }^{2}$ denotes the minimum $\chi^{2}$. These samples correspond to the $95 \%$ confidence level regions in any two dimensional plane of the model parameters when explaining the Higgs data (corresponding to be within $2 \sigma$ range).

In our calculations, the inputs parameters are taken as $m_{12}^{2}$, the physical Higgs masses $\left(m_{h}, m_{H}, m_{A}, m_{H^{ \pm}}\right)$, the vacuum expectation value ratio $(\tan \beta)$, the CP-even Higgs mixing angle $(\alpha)$, and the mixing angles of the down-type quark and charge lepton Yukawa couplings $\left(\theta_{d}, \theta_{l}\right)$. We fix respectively $m_{h}$ and $m_{H}$ as $125.5 \mathrm{GeV}$, and scan randomly the parameters in the following ranges:

$$
\begin{aligned}
& 50 \mathrm{GeV} \leq m_{A}, m_{H^{ \pm}} \leq 900 \mathrm{GeV}, \\
& -1 \leq \sin (\beta-\alpha) \leq 1, \quad 0.1 \leq \tan \beta \leq 50, \\
& 0 \leq \theta_{d} \leq \pi, \quad 0 \leq \theta_{l} \leq \pi, \\
& m_{12}^{2}\left(\mathrm{GeV}^{2}\right)= \pm(0.1)^{2}, \pm(1)^{2}, \pm(5)^{2}, \pm(10)^{2}, \pm(30)^{2}, \pm(50)^{2}, \\
& \pm(100)^{2}, \pm(180)^{2}, \pm(300)^{2}, \pm(400)^{2}, \pm(500)^{2}, \\
& \text { Scenario A : } m_{h}=125.5 \mathrm{GeV}, \quad 125.5 \mathrm{GeV} \leq m_{H} \leq 900 \mathrm{GeV} \text {, } \\
& \text { Scenario B : } m_{H}=125.5 \mathrm{GeV}, \quad 20 \mathrm{GeV} \leq m_{h} \leq 125.5 \mathrm{GeV} \text {. }
\end{aligned}
$$

HiggsSignals-1.1.0 automatically consider the effects of any neutral Higgs boson on $\chi^{2}$ if its mass satisfies

$$
\left|m_{h_{i}}-\hat{m}_{s}\right| \leq \Delta \hat{m}_{s} .
$$

Where $h_{i}$ denotes $h, H$ and $A . \hat{m}_{s}$ is the mass of signal $s$ and $\Delta \hat{m}_{s}$ is the experimental mass resolution of the analysis associated to signal $s$. However, if the $\chi^{2}$ contribution from the measured Higgs mass is activated, the combinations with a Higgs boson mass which does not fulfill eq. (3.2) are still considered. For the detailed introduction on the calculation of $\chi^{2}$, see $[47,48]$.

\section{Results and discussions}

\subsection{Scenario A}

Let us begin by discussing the scenario A in which the mass of the light CP-even Higgs $h$ is fixed as $125.5 \mathrm{GeV}$. In figure 1 , we project the surviving samples with $\chi^{2}$ being within $2 \sigma$ range on the planes of $\sin (\beta-\alpha)$ versus $m_{H}$ and $\sin (\beta-\alpha)$ versus $m_{A}$, respectively. The left panel shows that, for the heavy CP-even Higgs mass is close to $125.5 \mathrm{GeV}$, it can give the important contributions to $\chi^{2}$, and the absolute values of $\sin (\beta-\alpha)$ can be allowed to be as low as 0 , in which the $H V V$ couplings approach to SM while $h V V$ approach to 0 . For $m_{H} \geq 128 \mathrm{GeV}, \sin (\beta-\alpha)$ is allowed to be in the ranges of $0.83 \sim 1$ and $-1 \sim-0.89$. 

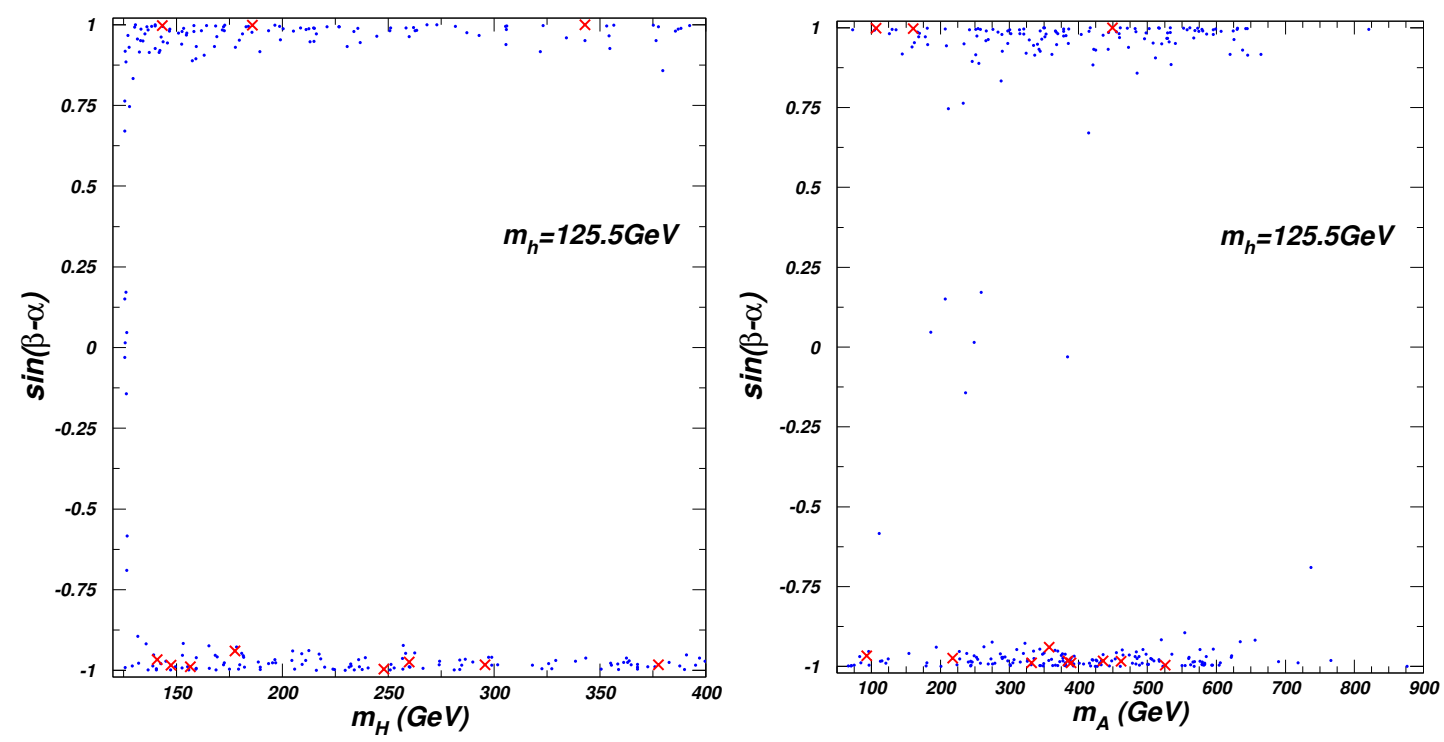

Figure 1. The scatter plots of surviving samples in scenario A projected on the planes of $\sin (\beta-\alpha)$ versus $m_{H}$ and $\sin (\beta-\alpha)$ versus $m_{A}$. The crosses (red), and bullets (blue) samples respectively have the values of $\chi^{2}$ in the ranges of $81.0 \sim 82.2$ and $82.2 \sim 87.2$, where the three values are respectively the minimal value of $\chi^{2}$ in scenario A $\left(\chi_{\text {Amin }}^{2}\right)$, the SM value $\left(\chi_{\mathrm{SM}}^{2}\right)$ and the value of $\chi^{2}$ at $2 \sigma$ level in scenario $\mathrm{A}\left(\chi_{A 2 \sigma}^{2}\right)$.
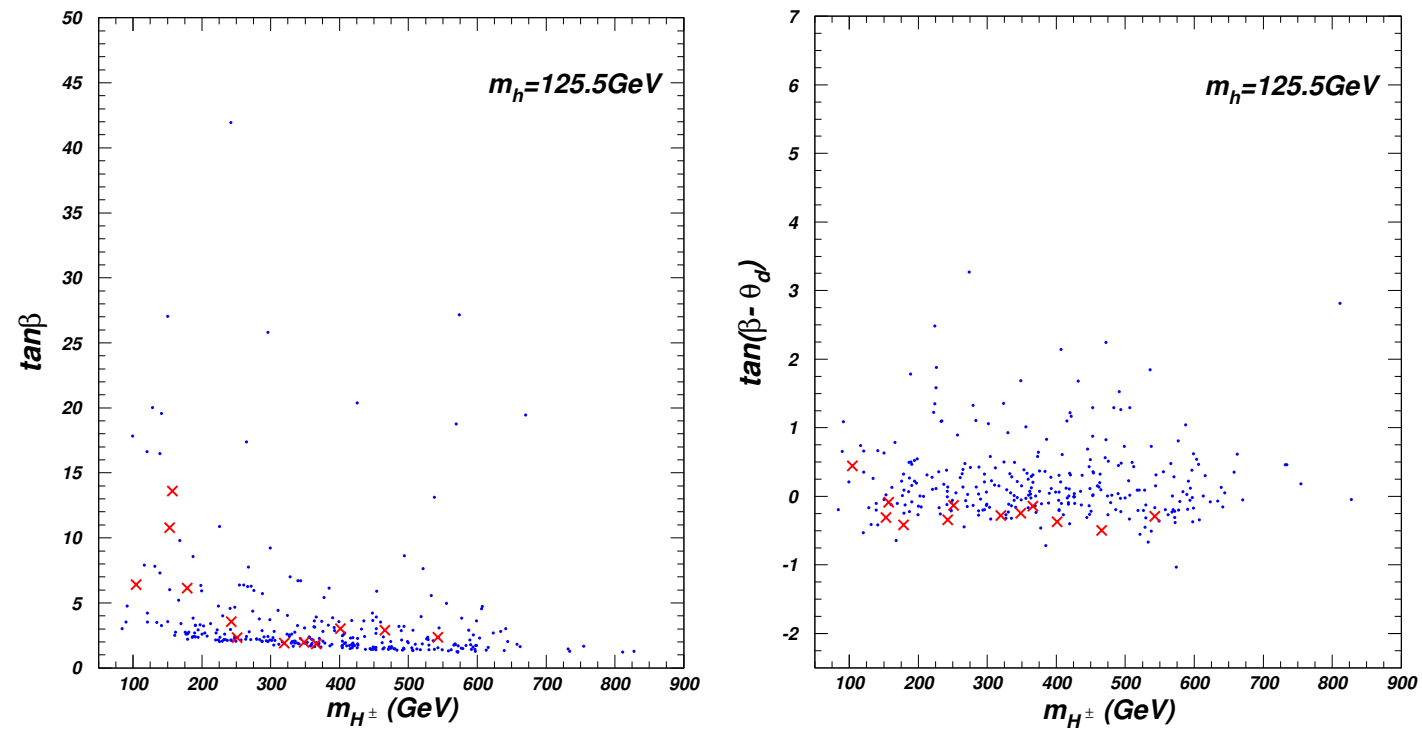

Figure 2. Same as figure 1 , but projected on the planes of $\tan \beta$ versus $m_{H^{ \pm}}$and $\tan \left(\beta-\theta_{d}\right)$ versus $m_{H^{ \pm}}$.

A small value of $\chi^{2}$ favors a large absolute value of $\sin (\beta-\alpha)$, which denotes that the absolute values of $h V V$ couplings approach to SM.

Unlike the heavy CP-even Higgs, the right panel of figure 1 shows that the CP-odd Higgs $A$ does not give the very visible effects on $\chi^{2}$ around $125.5 \mathrm{GeV}$ compared to the other mass ranges. $m_{A}$ is required to be larger than $63 \mathrm{GeV}$, and the on-shell decay $h \rightarrow A A$ is kinematically forbidden, which hardly affects the observed Higgs signals. 


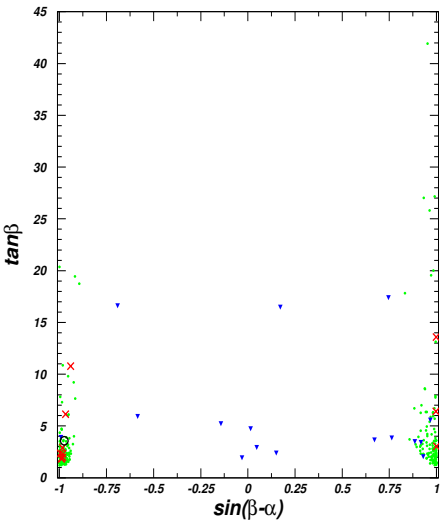

(a)

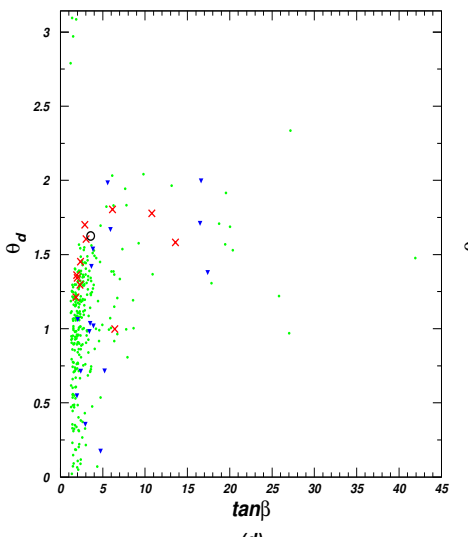

(d)

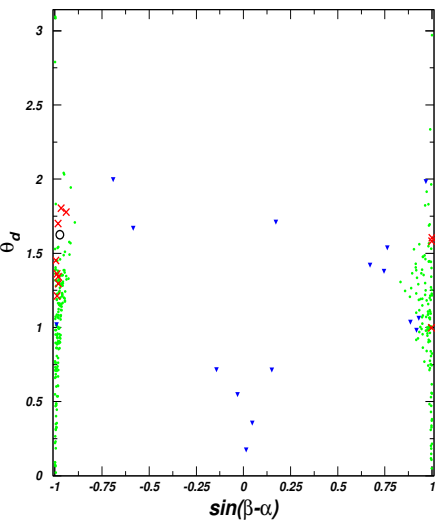

(b)

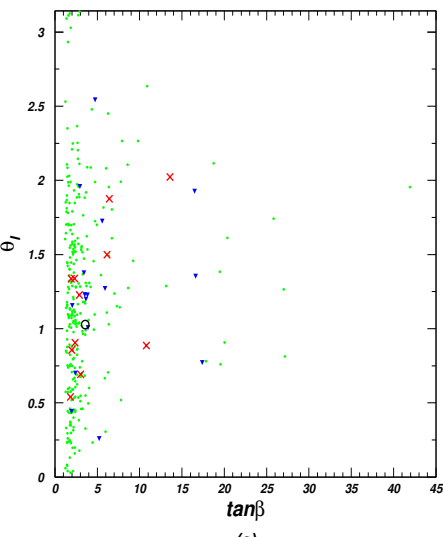

(e)

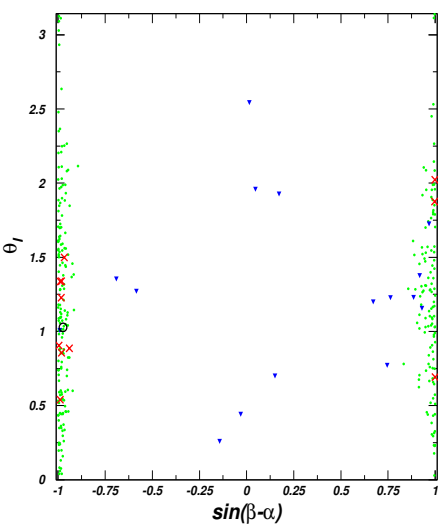

(c)

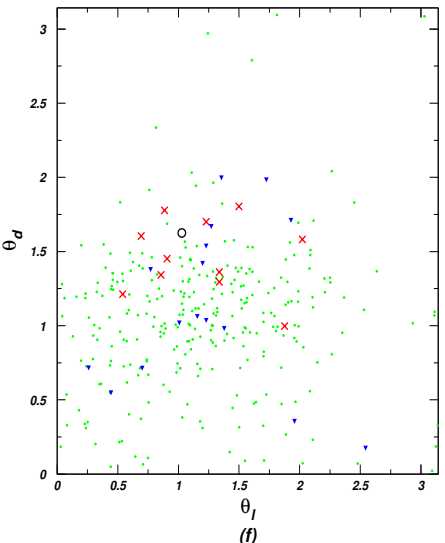

Figure 3. The scatter plots of surviving samples in scenario A projected on the planes of mixing angles. The $\chi^{2}$ values of the crosses (red), bullets (green) and inverted triangles (blue) samples are respectively in the ranges of $\chi_{A m i n}^{2} \sim \chi_{\mathrm{SM}}^{2}$ and $\chi_{\mathrm{SM}}^{2} \sim \chi_{A 2 \sigma}^{2}$ for $128 \mathrm{GeV} \leq m_{H} \leq 900 \mathrm{GeV}$, and $\chi_{\mathrm{SM}}^{2} \sim \chi_{A 2 \sigma}^{2}$ for $125.5 \mathrm{GeV} \leq m_{H}<128 \mathrm{GeV}$. The $\chi^{2}$ values of the circle (black) is $\chi_{\text {Amin }}^{2}$.
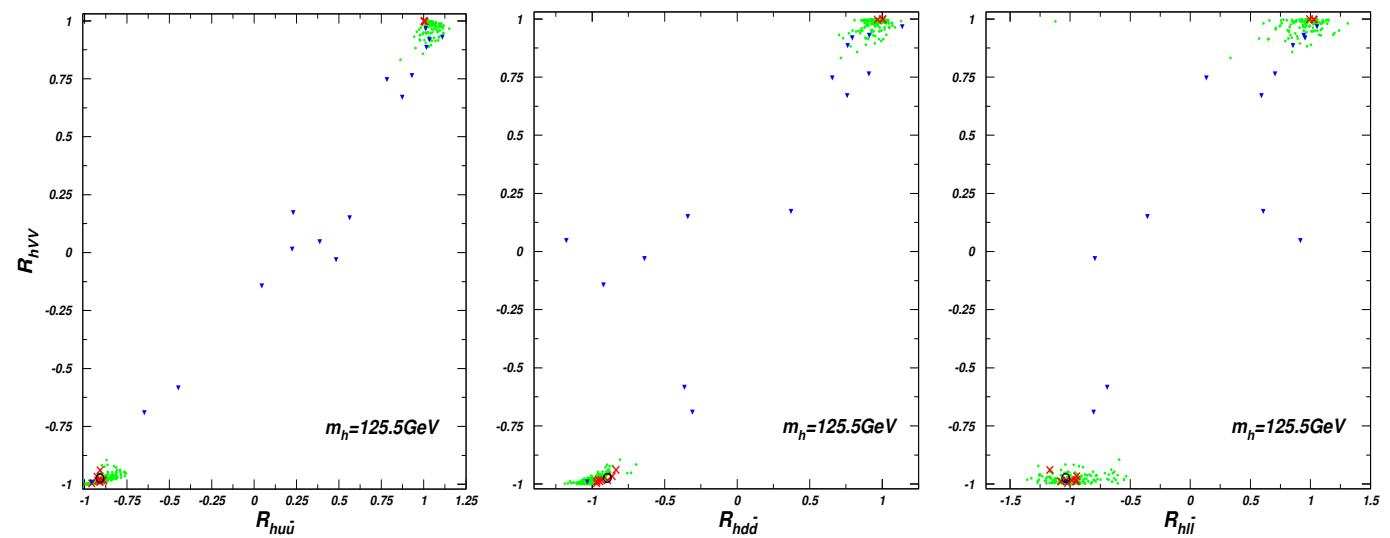

Figure 4. Same as figure 3, but projected on the planes of $R_{h V V}$ versus $R_{h u \bar{u}}, R_{h V V}$ versus $R_{h d \bar{d}}$ and $R_{h V V}$ versus $R_{h l \bar{l}}$. $R_{h V V}$ and $R_{h f \bar{f}}$ denote the light CP-even Higgs couplings to gauge bosons and $f \bar{f}(f=u, d, l)$ normalized to the SM couplings, respectively. 
In figure 2, the surviving samples are projected on the planes of $\tan \beta$ versus $m_{H^{ \pm}}$and $\tan (\beta-\alpha)$ versus $m_{H^{ \pm}}$. The left panel shows that the surviving samples favor $1<\tan \beta<5$ and allow $\tan \beta>30$ for $m_{H^{ \pm}}>230 \mathrm{GeV}$. The constraints from $\Delta m_{B_{d}}$ and $\Delta m_{B_{s}}$ require $\tan \beta$ to be larger than 1 for the whole range of $m_{H^{ \pm}}$, and larger than 3 for $m_{H^{ \pm}}<100 \mathrm{GeV}$. The right panel shows that the surviving samples favor $-0.5<\tan \left(\beta-\theta_{d}\right)<0.5$. The flavor interactions mediated by $H^{ \pm}$are proportional to $\tan \left(\beta-\theta_{d}\right)$. The constraints from the flavor observables allow $m_{H^{ \pm}}$to be smaller than $100 \mathrm{GeV}$ for the very small absolute of $\tan \left(\beta-\theta_{d}\right)$, and $\tan \left(\beta-\theta_{d}\right)$ to be larger than 3 for $m_{H^{ \pm}}>250 \mathrm{GeV}$. In addition, the samples with smaller $\chi^{2}$ than SM favor $\tan \left(\beta-\theta_{d}\right)$ to be in the range of $-0.5 \sim 0$ for $m_{H^{ \pm}}>150 \mathrm{GeV}$.

The contributions of the heavy CP-even Higgs boson to $\chi^{2}$ can be sizably suppressed for $m_{H} \geq 128 \mathrm{GeV}$. Therefore, we classify the surviving samples into groups: $125.5 \mathrm{GeV}$ $\leq m_{H}<128 \mathrm{GeV}$ and $128 \mathrm{GeV} \leq m_{H} \leq 900 \mathrm{GeV}$. In figure 3 , the two groups of surviving samples are projected on the planes of mixing angles $\left(\sin (\beta-\alpha), \tan \beta, \theta_{d}\right.$ and $\left.\theta_{l}\right)$. Figure 3 (a) shows that $\tan \beta$ can be over 20 for $\sin (\beta-\alpha)$ is close to 1 . Figure 3 (b) shows that, for $m_{H}>128 \mathrm{GeV}$, the mixing angle $\theta_{d}$ can loose constraints on $\sin (\beta-\alpha)$ visibly. For example, for $\theta_{d} \simeq 0$ (Type-II and Flipped $2 \mathrm{HDMs}$ ), the absolute value of $\sin (\beta-\alpha$ ) is required to be very close to 1 . While $\sin (\beta-\alpha)$ are allowed to vary in the range of $0.83 \sim 1$ and $-1 \sim-0.89$ for $\theta_{d}$ has the properly large value. Also figure 3 (c) shows that $\sin (\beta-\alpha)$ in the positive range is required to be very close to 1 for $\theta_{l} \simeq 0$ (Type-II and Lepton-specific $2 \mathrm{HDMs})$.

According to figures 3 (d) and (e), although the surviving samples favor a small value of $\tan \beta$, the value of $\chi^{2}$ can be smaller than SM for a large $\tan \beta$ when $\theta_{d}$ and $\theta_{l}$ have the proper large values, such as $\tan \beta=13.5, \theta_{d}=1.6$ and $\theta_{l}=2.0$.

Figure 3 (f) shows that the samples with smaller $\chi^{2}$ than SM are favored in the range of $1<\theta_{d}<2$ and $0.5<\theta_{l}<2.2$. Thus, it is possible that Type-I 2HDM gives the smaller value of $\chi^{2}$ than SM. The minimal value of $\chi^{2}(81.0)$ appears at $\theta_{d}=1.7$ and $\theta_{l}=1.3$.

In figure 4, the surviving samples are projected on the planes of Higgs couplings. For $125.5 \mathrm{GeV} \leq m_{H}<128 \mathrm{GeV}$, the heavy CP-even Higgs gives the important contributions to $\chi^{2}$. Therefore, there may be sizable deviations from SM for the couplings $h V V$, hu $\bar{u}$, $h d \bar{d}$ and $h l \bar{l}$. For $m_{H} \geq 128 \mathrm{GeV}$ and the $h V V$ coupling with the small absolute value, the $h b \bar{b}$ coupling by suppressed properly is required to obtain enough large $\operatorname{Br}\left(h \rightarrow Z Z^{*}\right)$ and $\operatorname{Br}(h \rightarrow \gamma \gamma)$. The $h \rightarrow \gamma \gamma$ and $h \rightarrow Z Z^{*} \rightarrow 4 l$ have the rather precise measurements and mass resolution, which play a very important role in the calculations of $\chi^{2}$. The signal strengths of $h \rightarrow \tau \tau$ have a large uncertainty and the signals are not important in the calculations of $\chi^{2}$. In addition, the mass resolution of $h \rightarrow \tau \tau$ is $20 \mathrm{GeV}$ for the analysis of ATLAS [54,55] and $25 \mathrm{GeV}$ for CMS [66], CDF [71] and D0 [72]. Therefore, $H$ and $A$ with $100 \sim 150 \mathrm{GeV}$ may contribute to $\chi^{2}$. The constraints on $h \tau \bar{\tau}$ is much more weaken than $h u \bar{u}$ and $h d \bar{d}$

For the samples with smaller $\chi^{2}$ than SM, there is the same sign for the light CP-even Higgs couplings to fermions and gauge bosons. Compared to SM, the $h V V$, hup and $h d \bar{d}$ couplings are suppressed, and the suppressions are allowed to be as low as 0.94, 0.90 and 0.83 , while the absolute value of $R_{h l \bar{l}}$ are allowed to be as high as 1.2 . 

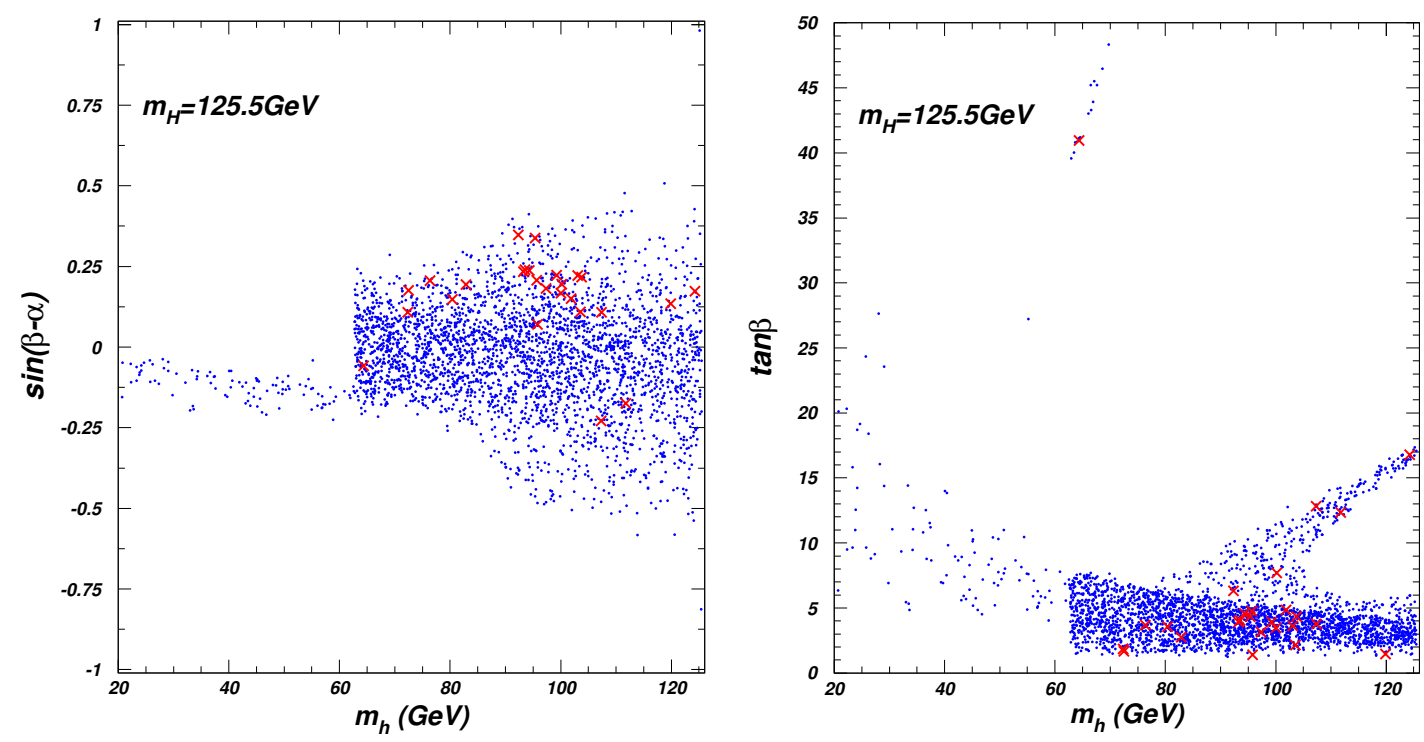

Figure 5. The scatter plots of surviving samples in scenario B projected on the planes of $\sin (\beta-\alpha)$ versus $m_{h}$ and $\tan \beta$ versus $m_{h}$. The crosses (red) and bullets (blue) samples respectively have the values of $\chi^{2}$ in the ranges of $81.5 \sim 82.2$ and $82.2 \sim 87.7$, where the three values are respectively the minimal value of $\chi^{2}$ in scenario $\mathrm{B}\left(\chi_{\mathrm{Bmin}}^{2}\right)$, the SM value $\left(\chi_{\mathrm{SM}}^{2}\right)$ and the value of $\chi^{2}$ at $2 \sigma$ level in scenario B $\left(\chi_{B 2 \sigma}^{2}\right)$.
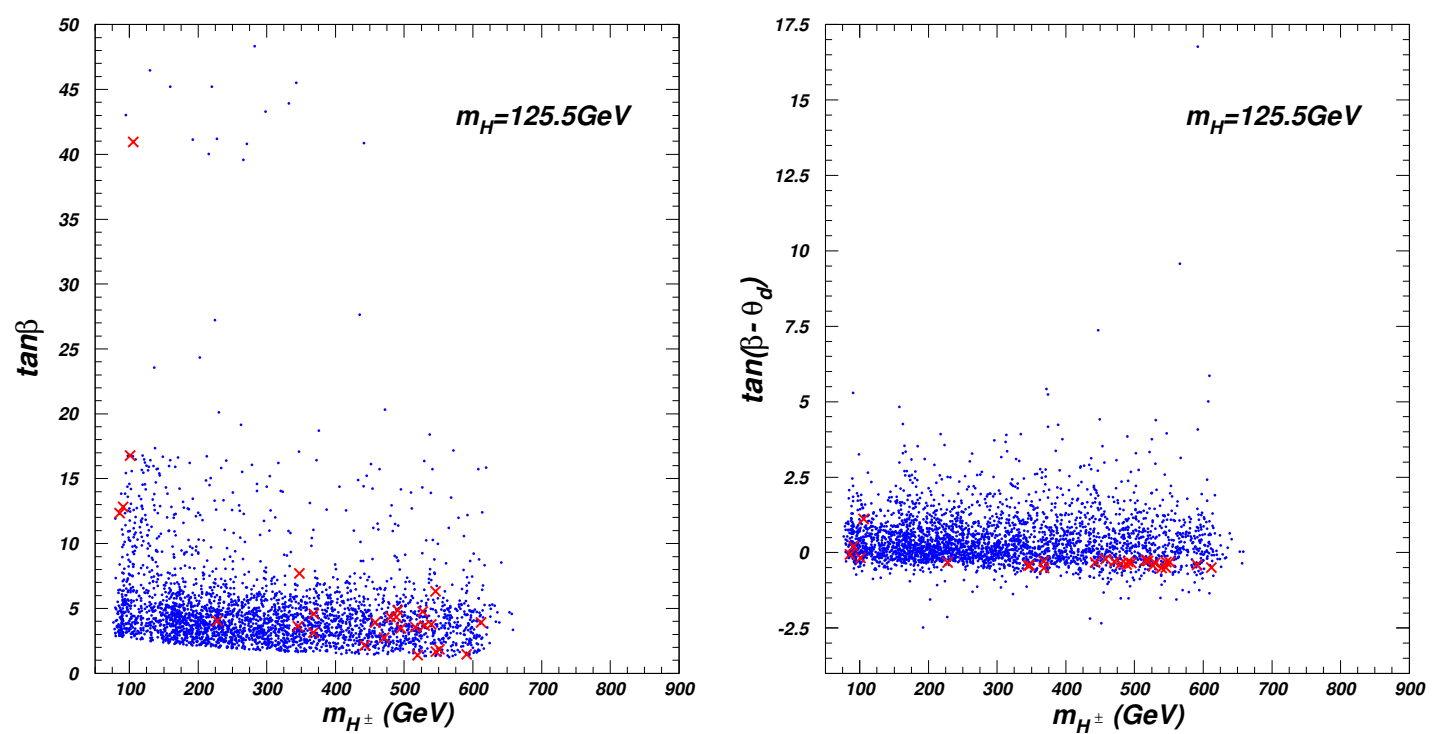

Figure 6. Same as figure 5, but projected on the planes of $\tan \beta$ versus $m_{H^{ \pm}}$and $\tan \left(\beta-\theta_{d}\right)$ versus $m_{H^{ \pm}}$.

\subsection{Scenario B}

Now we discuss the scenario B in which the mass of the heavy CP-even Higgs $H$ is fixed as $125.5 \mathrm{GeV}$. In figure 5 , we project the surviving samples with $\chi^{2}$ being within $2 \sigma$ range on the planes of $\sin (\beta-\alpha)$ versus $m_{h}$ and $\tan \beta$ versus $m_{h}$, respectively. The left panel shows that, for $125 \mathrm{GeV} \leq m_{h} \leq 125.5 \mathrm{GeV}$, the absolute values of $\sin (\beta-\alpha)$ can be allowed 

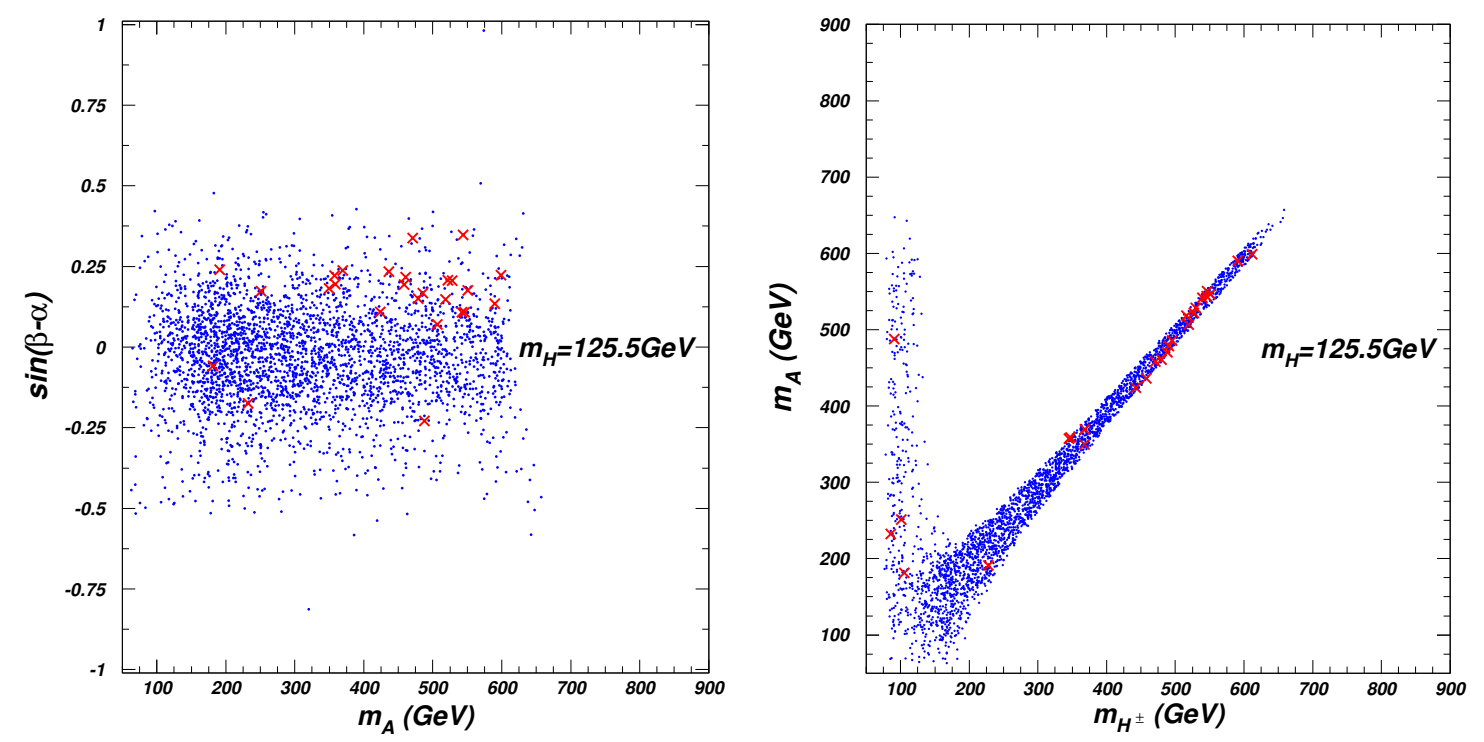

Figure 7. Same as figure 5, but projected on the planes of $\sin (\beta-\alpha)$ versus $m_{A}$ and $m_{A}$ versus $m_{H \pm}$.

to be as high as 1 , which denotes $h V V$ couplings approach to SM while $H V V$ approach to 0 . Such light CP-even Higgs can give the important contributions to $\chi^{2}$. The minimal absolute value of $\sin (\beta-\alpha)$ decreases with $m_{h}$ in principle. The light CP-even Higgs can be allowed to be as low as $20 \mathrm{GeV}$ for $-0.25<\sin (\beta-\alpha) \leq 0$. To be consistent with LEP constraints, the suppression of $h b \bar{b}$ coupling is also required for some surviving samples in addition to the small absolute value of $\sin (\beta-\alpha)$. In addition, the small values of $\chi^{2}$ favor $-0.25<\sin (\beta-\alpha)<0.38$, which denotes that the absolute values of $H V V$ couplings are close to SM. The right panel shows that $\tan \beta$ is required to be larger than 4 for $m_{h}<$ $60 \mathrm{GeV}$, which is due to the constraints of the observed Higgs signals on the opening decay $H \rightarrow h h$.

In figure 6 , the surviving samples are projected on the planes of $\tan \beta$ versus $m_{H^{ \pm}}$and $\tan \left(\beta-\theta_{d}\right)$ versus $m_{H^{ \pm}}$. The left panel shows that the surviving samples favor $1<\tan \beta<$ 7 and allow $\tan \beta>40$ for the proper $m_{H^{ \pm}}$. Similar to scenario A, $\tan \beta$ is required to be larger than 1 for the whole range of $m_{H^{ \pm}}$, and larger than 3 for the $m_{H^{ \pm}}<100 \mathrm{GeV}$. The right panel shows that the surviving samples favor $-1<\tan \left(\beta-\theta_{d}\right)<2.5$. The constraints from the flavor observables require the absolute value of $\tan \left(\beta-\theta_{d}\right)$ to be smaller than 2.5 for $m_{H^{ \pm}}<100 \mathrm{GeV}$, and allow $\tan \left(\beta-\theta_{d}\right)$ to be larger than 10 for $m_{H^{ \pm}}>600 \mathrm{GeV}$. The samples with smaller $\chi^{2}$ than SM favor $\tan \left(\beta-\theta_{d}\right)$ to be in the range of $-0.5 \sim 0$ for the large $m_{H^{ \pm}}$and be enhanced for $m_{H^{ \pm}}$around $100 \mathrm{GeV}$.

In figure 7 , the surviving samples are projected on the planes of $\sin (\beta-\alpha)$ versus $m_{A}$ and $m_{A}$ versus $m_{H^{ \pm}}$. Similar to scenario A, the CP-odd Higgs $A$ does not give the very visible effects on the $\chi^{2}$ around $125.5 \mathrm{GeV}$ compared to the other mass ranges. The on-shell decay $H \rightarrow A A$ is kinematically forbidden, which hardly affects the observed Higgs signals. The right panel shows that most of samples lie in the region where there is small mass difference between $m_{A}$ and $m_{H^{ \pm}}$, and some other samples lie in the small region 


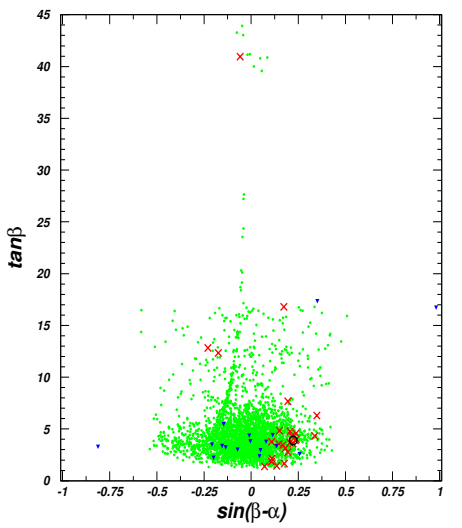

(a)

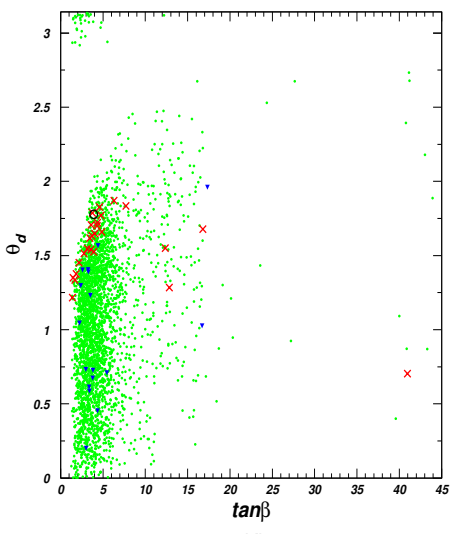

(d)

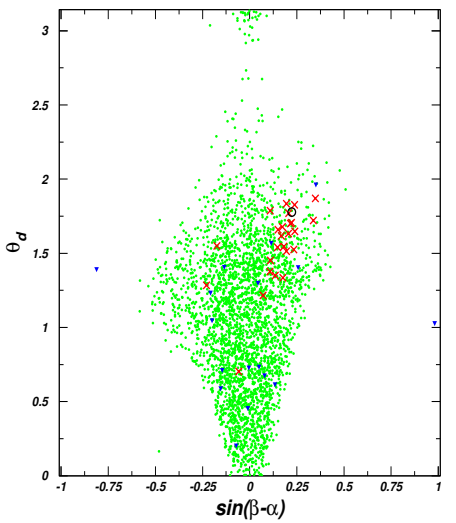

(b)

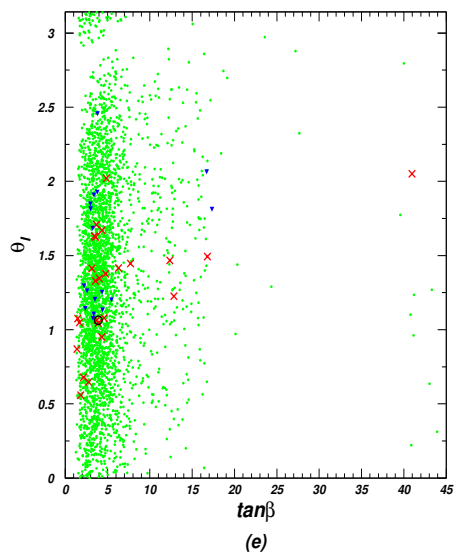

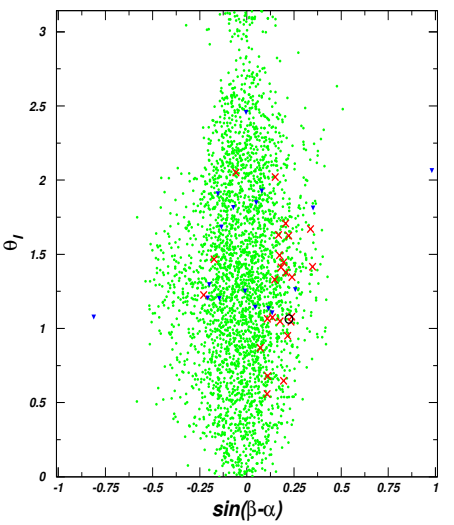

(c)

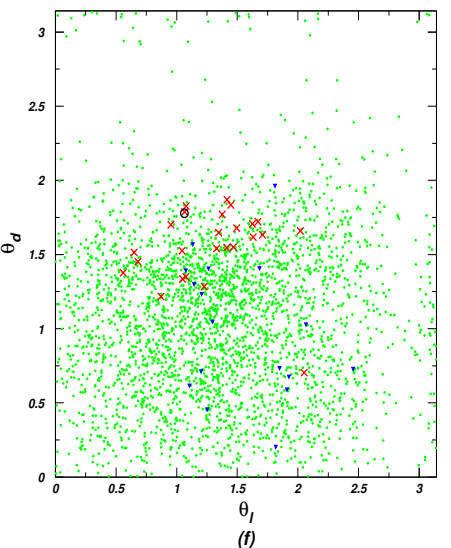

Figure 8. The scatter plots of surviving samples in scenario B projected on the planes of mixing angles. The $\chi^{2}$ values of the crosses (red), bullets (green) and inverted triangles (blue) samples are respectively in the ranges of $\chi_{\mathrm{Bmin}}^{2} \sim \chi_{\mathrm{SM}}^{2}$ and $\chi_{\mathrm{SM}}^{2} \sim \chi_{B 2 \sigma}^{2}$ for $20 \mathrm{GeV} \leq m_{H}<125 \mathrm{GeV}$, and $\chi_{\mathrm{SM}}^{2} \sim \chi_{B 2 \sigma}^{2}$ for $125 \mathrm{GeV} \leq m_{H}<125.5 \mathrm{GeV}$. The $\chi^{2}$ values of the circle (black) is $\chi_{\mathrm{Bmin}}^{2}$.
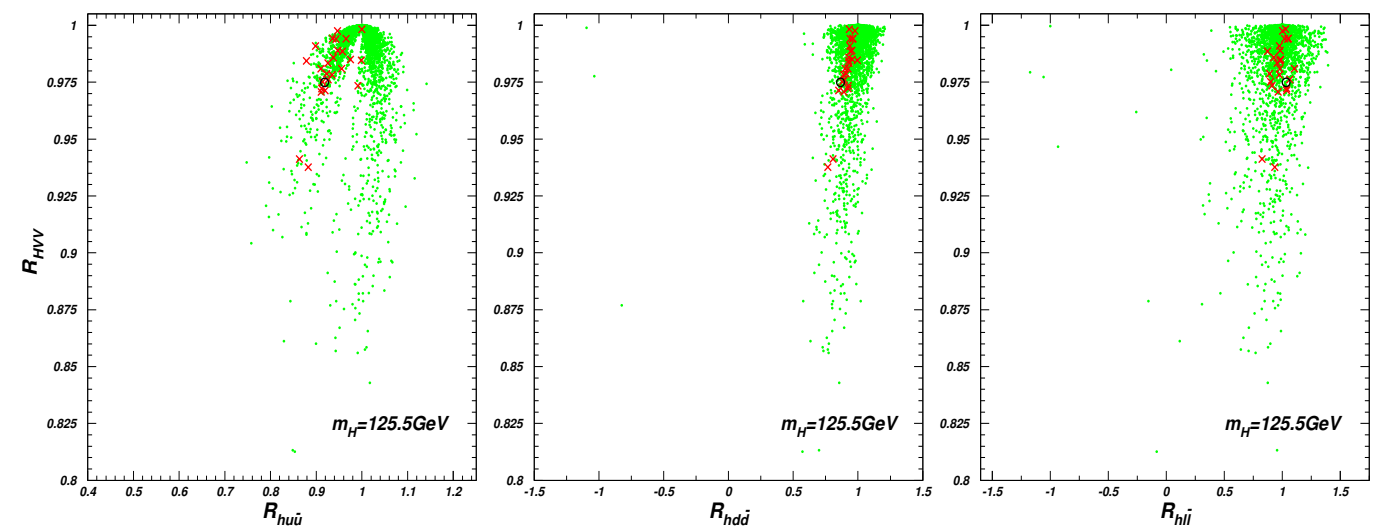

Figure 9. Same as figure 8, but only the samples with $20 \mathrm{GeV} \leq m_{h}<120 \mathrm{GeV}$ projected on the planes of $R_{H V V}$ versus $R_{H u \bar{u}}, R_{H V V}$ versus $R_{H d \bar{d}}$ and $R_{H V V}$ versus $R_{H l \bar{l}}$. $R_{H V V}$ and $R_{H f \bar{f}}$ denote the heavy CP-even Higgs couplings to gauge bosons and $f \bar{f}(f=u, d, l)$ normalized to the SM couplings, respectively. 
where $m_{H^{ \pm}}$is around $100 \mathrm{GeV}$ and has large mass difference from $m_{A}$. Assuming $m_{12}^{2}=0$, Baradhwaj Coleppa et al. have shown the strong correlations between $m_{A}$ and $m_{H^{ \pm}}$in the Type-II $2 \mathrm{HDM}$ [16]. Here $m_{12}^{2}$ is taken as various values, the strong correlations still exist but the latter region becomes slightly wider than [16]. The main reason is from the constraints of $\Delta \rho$, which is also studied in detail in [73]. Since there is small mass difference between $m_{h}$ and $m_{H}$ for the scenario $\mathrm{B}, m_{A}$ and $m_{H^{ \pm}}$should have the small mass difference to cancel the contributions of $m_{h}$ and $m_{H}$ to $\Delta \rho$. However, for $m_{H^{ \pm}}$ is around $m_{H}$, the contributions to $\Delta \rho$ from $\left(m_{h}, m_{H^{ \pm}}\right)$and $\left(m_{A}, m_{H^{ \pm}}\right)$loops can be canceled by the $\left(m_{h}, m_{H}\right)$ and $\left(m_{A}, m_{H}\right)$ loops. Thus $m_{A}$ is allowed to vary from $70 \mathrm{GeV}$ to $700 \mathrm{GeV}$ for $m_{H^{ \pm}}$around $100 \mathrm{GeV}$.

The contributions of the light CP-even Higgs boson to $\chi^{2}$ can be sizably suppressed for $m_{h}<125 \mathrm{GeV}$. Therefore, we classify the surviving samples into groups: $20 \mathrm{GeV} \leq m_{H}<$ $125 \mathrm{GeV}$ and $125 \mathrm{GeV} \leq m_{H} \leq 125.5 \mathrm{GeV}$. In figure 8, the two groups of surviving samples are projected on the planes of mixing angles. Figure 8 (a) shows that the samples with $\tan \beta>20$ require $\sin (\beta-\alpha)$ to approach to 0 . Figure 8 (b) shows that, for $m_{h}<125 \mathrm{GeV}$, $\theta_{d}$ can loose the constraints on $\sin (\beta-\alpha)$ sizably. For example, for $\theta_{d} \simeq 0$ (Type-II and Flipped $2 \mathrm{HDMs}$ ), $\sin (\beta-\alpha)$ is allowed to vary from -0.1 to 0.06 . While for $\theta_{d} \simeq \frac{\pi}{2}$ (Type-I and Lepton-specific $2 \mathrm{HDMs}), \sin (\beta-\alpha)$ is allowed to vary in the range of $-0.5 \sim 0.44$. Further, figure 8 (c) shows that $\theta_{l} \simeq 0$ (Type-II and Lepton-specific $2 \mathrm{HDMs}$ ) also gives the strong constraints on $\sin (\beta-\alpha),-0.18 \leq \sin (\beta-\alpha) \leq 0.12$.

Similar to scenario A, figures 8 (d) and (e) show that, although the surviving samples favor $1<\tan \beta<7$, the value of $\chi^{2}$ can be smaller than SM for a large $\tan \beta$ when $\theta_{d}$ and $\theta_{l}$ have the proper large values. Even for $\tan \beta=41$, the value of $\chi^{2}$ can be smaller than SM for $\theta_{d}=0.7$ and $\theta_{l}=2.1$. Figure 8 (f) shows that the samples with smaller than $\mathrm{SM}$ are in the range of $0.5<\theta_{d}<2$ and $0.5<\theta_{l}<2.2$. The minimal value of $\chi^{2}(81.5)$ appears at $\theta_{d}=1.8$ and $\theta_{l}=1.1$.

In figure 9 , the surviving samples with $20 \mathrm{GeV} \leq m_{h}<125 \mathrm{GeV}$ are projected on the planes of Higgs couplings. Similar to scenario A, for the $H V V$ coupling with the small absolute value, the $H b \bar{b}$ coupling by suppressed properly is required to obtain enough large $\operatorname{Br}\left(h \rightarrow Z Z^{*}\right)$ and $\operatorname{Br}(h \rightarrow \gamma \gamma)$. The constraints on $h \tau \bar{\tau}$ is much more weaken than $h u \bar{u}$ and $h d \bar{d}$. For the samples with smaller $\chi^{2}$ than SM, there is the same sign for the heavy CP-even Higgs couplings to fermions and gauge bosons. Compared to SM, the $H V V$, $H u \bar{u}$ and $H d \bar{d}$ couplings are suppressed, and the suppressions are allowed to be as low as 0.94, 0.86 and 0.77 , respectively. However, the $H l \bar{l}$ coupling can be allowed to have a $10 \%$ enhancement, or $17 \%$ suppression.

In table 3 we present the detailed information for the four samples with the minimal values of $\chi^{2}$ in the scenario $\mathrm{A}\left(125.5 \mathrm{GeV} \leq m_{H}<128 \mathrm{GeV}\right.$ and $\left.128 \mathrm{GeV} \leq m_{H} \leq 900 \mathrm{GeV}\right)$ and scenario $\mathrm{B}\left(20 \mathrm{GeV} \leq m_{h}<125 \mathrm{GeV}\right.$ and $\left.125 \mathrm{GeV} \leq m_{h} \leq 125.5 \mathrm{GeV}\right)$. For the four cases, $\theta_{d}$ and $\theta_{l}$ of the samples with the minimal $\chi^{2}$ are in the ranges of $1.5 \sim 1.8$ and $1.0 \sim 2.0$. For the scenario A with $128 \mathrm{GeV} \leq m_{H} \leq 900 \mathrm{GeV}$ and scenario B with $20 \mathrm{GeV}$ $\leq m_{h}<125 \mathrm{GeV}$, the absolute values for the $125.5 \mathrm{GeV}$ Higgs couplings to $V V$ approach to SM, and the couplings to $u \bar{u}$ and $d \bar{d}$ have around $10 \%$ suppressions compared to SM. The minimal $\chi^{2}$ values of the two cases are respectively 81.0 and 81.5 , which are marginally 


\begin{tabular}{|c|c|c|c|c|}
\hline & scenario A & scenario A & scenario B & scenario B \\
\hline$m_{h}(\mathrm{GeV})$ & 125.5 & 125.5 & 99.3 & 125.4 \\
$m_{H}(\mathrm{GeV})$ & 126.1 & 259.9 & 125.5 & 125.5 \\
$m_{A}(\mathrm{GeV})$ & 258.9 & 217.4 & 598.8 & 342.3 \\
$m_{H^{ \pm}}(\mathrm{GeV})$ & 139.1 & 242.8 & 612.1 & 347.1 \\
\hline$m_{12}^{2}(\mathrm{GeV})$ & 900 & 10000 & 0.01 & 900 \\
$\sin (\beta-\alpha)$ & 0.172 & -0.973 & 0.222 & -0.042 \\
$\tan \beta$ & 16.48 & 3.57 & 3.91 & 17.07 \\
$\theta_{d}$ & 1.71 & 1.63 & 1.78 & 1.53 \\
$\theta_{l}$ & 1.93 & 1.03 & 1.06 & 1.30 \\
\hline$\chi^{2}$ & 83.3 & 81.0 & 81.5 & 83.0 \\
\hline$R_{h V V}$ & 0.172 & -0.973 & 0.222 & -0.042 \\
$R_{h u \bar{u}}$ & 0.231 & -0.909 & 0.472 & 0.016 \\
$R_{h d \bar{d}}$ & 0.371 & -0.895 & 0.702 & -0.020 \\
$R_{h l \bar{l}}$ & 0.608 & -1.04 & -0.033 & -0.260 \\
\hline$R_{H V V}$ & 0.985 & 0.229 & 0.975 & 0.999 \\
$R_{H u \bar{u}}$ & 0.975 & 0.502 & 0.918 & 1.002 \\
$R_{H d \bar{d}}$ & 0.950 & 0.561 & 0.866 & 1.000 \\
$R_{H l \bar{l}}$ & 0.909 & -0.040 & 1.033 & 0.990 \\
\hline$R_{A u \bar{u}}$ & -0.061 & -0.280 & -0.256 & -0.059 \\
$R_{A d \bar{d}}$ & 0.202 & 0.341 & 0.491 & 0.022 \\
$R_{A l \bar{l}}$ & 0.443 & -0.277 & -0.262 & -0.217 \\
\hline & & & & \\
\hline & & & +19 & \\
\hline
\end{tabular}

Table 3. The detailed information of the four samples with the minimal values of $\chi^{2}$ in the scenario A $\left(125.5 \mathrm{GeV} \leq m_{H}<128 \mathrm{GeV}\right.$ and $\left.128 \mathrm{GeV} \leq m_{H} \leq 900 \mathrm{GeV}\right)$ and scenario $\mathrm{B}(20 \mathrm{GeV}$ $\leq m_{h}<125 \mathrm{GeV}$ and $\left.125 \mathrm{GeV} \leq m_{h} \leq 125.5 \mathrm{GeV}\right)$. Where $R_{A u \bar{u}}, R_{A d \bar{d}}$ and $R_{A l \bar{l}}$ are from the interactions, $\frac{m_{f}}{v} R_{A f \bar{f}} A \bar{f} \gamma^{5} f$ with $f=u, d, l$.

smaller than SM value (82.2). This implies that the A2HDM can provide marginally better fit to the observed Higgs signals than SM at the expense of additional parameters. Similarly, the minimal dilaton model can not provide much better fit to LHC and Tevatron Higgs data than SM [74]. The fit given by little Higgs models at most approaches to SM for very large scale $f$ [75-77], while Next-to-Minimal Supersymmetric Standard Model [78-80] can give much better fit than SM.

After Moriond 2013, the CMS diphoton data has changed drastically, which is no longer enhanced. In addition to the four typical $2 \mathrm{HDMs}$, the Higgs data after Moriond 2013 have been used to examine the A2HDM in refs. [23, 24, 30, 32]. Refs. [32] assumes the both Higgs doublet fields $\left(\Phi_{1}\right.$ and $\left.\Phi_{2}\right)$ to couple to the up-type quarks, down-type quarks and charged leptons with aligned Yukawa matrices. However, refs. [23, 24, 30] and this 
paper use a freedom to eliminate the coupling of up-type quarks to $\Phi_{1}$. In our discussions, we consider more relevant theoretical and experimental constraints than refs. [23, 24, 30]. Our paper shows that the theoretical constraint from perturbativity disfavors a large $\tan \beta$ much more visibly than ref. [24]. In our analysis, we consider the 73 Higgs signal strengths observables from ATLAS, CMS, CDF and D0 collaborations as well as the four Higgs mass measurements from ATLAS and CMS, which are more than refs. [23, 24, 30]. The HiggsSignals-1.1.0 is employed to takes into account the signal efficiencies, experimental mass resolution and uncertainties. Our paper shows that the Higgs couplings to gauge bosons and fermions are not more strongly constrained than refs. [23, 24, 30, 32]. Refs. [23, $30]$ focus on the constraints of the Higgs signals on the Higgs couplings to gauge bosons and fermions. In addition to these Higgs couplings, we also give the allowed parameters spaces in detail, including $\tan \beta, \sin (\beta-\alpha), \theta_{d}, \theta_{l}$, the neutral and charged Higgs masses, and show explicitly that the proper $\theta_{d}$ can loose the constraints on $\sin (\beta-\alpha), \tan \beta$ and $m_{H^{ \pm}}$sizably. An interesting finding is that when $\theta_{d}$ and $\theta_{l}$ have the proper large values, the value of $\chi^{2}$ can be smaller than SM for a large $\tan \beta$ (even $\tan \beta=41$ ), although the $2 \sigma$ Higgs data and the relevant theoretical and experimental constraints favor a small tan $\beta$.

\section{Conclusion}

In this note, we studied the implications of the latest Higgs signals on a two-Higgs-doublet model with the alignment of the down-type quarks and charged lepton Yukawa coupling matrices. In our analysis, we consider the theoretical constraints from vacuum stability, unitarity and perturbativity as well as the experimental constraints from the electroweak precision data, flavor observables and the non-observation of additional Higgs at collider. We obtained the following observations:

(i) In the scenario $\mathrm{A}\left(m_{h}\right.$ is fixed as $\left.125.5 \mathrm{GeV}\right), \sin (\beta-\alpha)$ is allowed to be in the range of $-1 \sim 1$ for $125.5 \mathrm{GeV} \leq m_{H}<128 \mathrm{GeV}$. For $m_{H} \geq 128 \mathrm{GeV}, \sin (\beta-\alpha)$ is allowed to be in the ranges of $0.83 \sim 1$ and $-1 \sim-0.89$ for the proper $\theta_{d}$, but be very close to 1 or -1 for $\theta_{d}=0$. Also, the mixing angle $\theta_{d}$ can loose the constraints on $\tan \beta$ and $m_{H^{ \pm}}$sizably. Although the surviving samples favor $1<\tan \beta<5$, the value of $\chi^{2}$ can be smaller than SM for a large $\tan \beta$ when $\theta_{d}$ and $\theta_{l}$ have the proper large values. $m_{H^{ \pm}}$is allowed to be below $100 \mathrm{GeV}$ for the absolute value of $\tan \left(\beta-\theta_{d}\right)$ is very small, and the samples with the smaller $\chi^{2} \operatorname{than} \mathrm{SM}$ favor $0.5<\tan \left(\beta-\theta_{d}\right)<$ 0 for $m_{H^{ \pm}}>150 \mathrm{GeV}$.

(ii) In the scenario $\mathrm{B}\left(m_{H}\right.$ is fixed as $\left.125.5 \mathrm{GeV}\right), \sin (\beta-\alpha)$ is allowed to be in the range of $-1 \sim 1$ for $125 \mathrm{GeV} \leq m_{h} \leq 125.5 \mathrm{GeV}$, and the minimal absolute value of $\sin (\beta-\alpha)$ decreases with $m_{h}$ in principle. The light CP-even Higgs can be allowed to be as low as $20 \mathrm{GeV}$ for $-0.25<\sin (\beta-\alpha) \leq 0$. The constraints of the observed Higgs signals on the opening decay $H \rightarrow h h$ require $\tan \beta$ to be larger than 4 for $m_{h}<60 \mathrm{GeV}$. Similar to scenario A, the mixing angle $\theta_{d}$ can loose the constraints on $\sin (\beta-\alpha), \tan \beta$ and $m_{H^{ \pm}}$sizably. For $m_{h}<125 \mathrm{GeV}, \theta_{d}$ around $\frac{\pi}{2}$ can allow $\sin (\beta-\alpha)$ to be in the range of $-0.5 \sim 0.44$. Although the surviving samples favor 1 
$<\tan \beta<7$, the value of $\chi^{2}$ can be smaller than SM for $\tan \beta>40$ when $\theta_{d}$ and $\theta_{l}$ have the proper large values. $m_{H^{ \pm}}$is allowed to be below $100 \mathrm{GeV}$ for the absolute value of $\tan \left(\beta-\theta_{d}\right)$ is smaller than 2.5 , and the samples with the smaller $\chi^{2}$ than SM favor $-0.5<\tan \left(\beta-\theta_{d}\right)<0$ for the large $m_{H^{ \pm}}$.

(iii) The model can provide the marginally better fit to available Higgs signals data than SM. For $m_{h}=125.5 \mathrm{GeV}$, the absolute values of $h V V$, $h u \bar{u}$ and $h d \bar{d}$ couplings are respectively allowed to be as low as $0.94,0.90$ and 0.83 , and $\theta_{d}$ and $\theta_{l}$ are favored in the ranges of $1 \sim 2$ and $0.5 \sim 2.2$. For $m_{H}=125.5 \mathrm{GeV}$, the $H V V, H u \bar{u}$ and $H d \bar{d}$ couplings are respectively allowed to be as low as $0.94,0.86$ and 0.77 , and $\theta_{d}$ and $\theta_{l}$ are favored in the ranges of $0.5 \sim 2$ and $0.5 \sim 2.2$.

\section{Acknowledgments}

We would like to thank Nazila Mahmoudi, Johan Rathsman and Oscar Stål for helpful discussions on the codes SuperIso, 2HDMC and HiggsBounds. This work was supported by the National Natural Science Foundation of China (NNSFC) under grant Nos. 11005089, 11105116 and 11175151.

Open Access. This article is distributed under the terms of the Creative Commons Attribution License (CC-BY 4.0), which permits any use, distribution and reproduction in any medium, provided the original author(s) and source are credited.

\section{References}

[1] CMS collaboration, Observation of a new boson at a mass of $125 \mathrm{GeV}$ with the CMS experiment at the LHC, Phys. Lett. B 716 (2012) 30 [arXiv:1207.7235] [INSPIRE].

[2] ATLAS collaboration, Observation of a new particle in the search for the standard model Higgs boson with the ATLAS detector at the LHC, Phys. Lett. B 716 (2012) 1 [arXiv: 1207.7214] [INSPIRE].

[3] CDF, D0 collaboration, T. Aaltonen et al., Higgs boson studies at the Tevatron, Phys. Rev. D 88 (2013) 052014 [arXiv: 1303.6346] [INSPIRE].

[4] T. Lee, A theory of spontaneous T violation, Phys. Rev. D 8 (1973) 1226 [INSPIRE].

[5] H. Haber, G.L. Kane and T. Sterling, The fermion mass scale and possible effects of Higgs bosons on experimental observables, Nucl. Phys. B 161 (1979) 493 [INSPIRE].

[6] L.J. Hall and M.B. Wise, Flavor changing Higgs-boson couplings, Nucl. Phys. B 187 (1981) 397 [inSPIRE].

[7] J.F. Donoghue and L.F. Li, Properties of charged Higgs bosons, Phys. Rev. D 19 (1979) 945 [INSPIRE].

[8] V.D. Barger, J. Hewett and R. Phillips, New constraints on the charged Higgs sector in two Higgs doublet models, Phys. Rev. D 41 (1990) 3421 [INSPIRE].

[9] J. Bagger, E. Poppitz and L. Randall, The $R$ axion from dynamical supersymmetry breaking, Nucl. Phys. B 426 (1994) 3 [hep-ph/9405345] [INSPIRE]. 
[10] A. Akeroyd and W.J. Stirling, Light charged Higgs scalars at high-energy $e^{+} e^{-}$colliders, Nucl. Phys. B 447 (1995) 3 [INSPIRE].

[11] A. Akeroyd, Nonminimal neutral Higgs bosons at LEP-2, Phys. Lett. B 377 (1996) 95 [hep-ph/9603445] [INSPIRE].

[12] A.G. Akeroyd, Fermiophobic and other nonminimal neutral Higgs bosons at the LHC, J. Phys. G 24 (1998) 1983 [hep-ph/9803324] [INSPIRE].

[13] M. Aoki, S. Kanemura, K. Tsumura and K. Yagyu, Models of Yukawa interaction in the two Higgs doublet model and their collider phenomenology, Phys. Rev. D 80 (2009) 015017 [arXiv: 0902.4665] [INSPIRE].

[14] C.-Y. Chen and S. Dawson, Exploring two Higgs doublet models through Higgs production, Phys. Rev. D 87 (2013) 055016 [arXiv: 1301.0309] [INSPIRE].

[15] B. Grinstein and P. Uttayarat, Carving out parameter space in type-II two Higgs doublets model, JHEP 06 (2013) 094 [Erratum ibid. 1309 (2013) 110] [arXiv:1304. 0028] [INSPIRE].

[16] B. Coleppa, F. Kling and S. Su, Constraining type II 2HDM in light of LHC Higgs searches, JHEP 01 (2014) 161 [arXiv:1305.0002] [INSPIRE].

[17] O. Eberhardt, U. Nierste and M. Wiebusch, Status of the two-Higgs-doublet model of type-II, JHEP 07 (2013) 118 [arXiv:1305.1649] [INSPIRE].

[18] C.-W. Chiang and K. Yagyu, Implications of Higgs boson search data on the two-Higgs doublet models with a softly broken $Z_{2}$ symmetry, JHEP 07 (2013) 160 [arXiv:1303.0168] [INSPIRE].

[19] C.-Y. Chen, S. Dawson and M. Sher, Heavy Higgs searches and constraints on two Higgs doublet models, Phys. Rev. D 88 (2013) 015018 [arXiv: 1305.1624] [INSPIRE].

[20] L. Wang and X.-F. Han, LHC diphoton Higgs signal and top quark forward-backward asymmetry in quasi-inert Higgs doublet model, JHEP 05 (2012) 088 [arXiv: 1203.4477] [INSPIRE].

[21] N. Craig, J. Galloway and S. Thomas, Searching for signs of the second Higgs doublet, arXiv:1305.2424 [INSPIRE].

[22] G. Bélanger, B. Dumont, U. Ellwanger, J. Gunion and S. Kraml, Global fit to Higgs signal strengths and couplings and implications for extended Higgs sectors, Phys. Rev. D 88 (2013) 075008 [arXiv:1306.2941] [INSPIRE].

[23] V. Barger, L.L. Everett, H.E. Logan and G. Shaughnessy, Scrutinizing h(125) in two Higgs doublet models at the LHC, ILC and Muon Collider, Phys. Rev. D 88 (2013) 115003 [arXiv: 1308.0052] [INSPIRE].

[24] D. López-Val, T. Plehn and M. Rauch, Measuring extended Higgs sectors as a consistent free couplings model, JHEP 10 (2013) 134 [arXiv:1308.1979] [INSPIRE].

[25] S. Choi, S. Jung and P. Ko, Implications of LHC data on $125 \mathrm{GeV}$ Higgs-like boson for the standard model and its various extensions, JHEP 10 (2013) 225 [arXiv:1307.3948] [INSPIRE].

[26] S. Chang et al., Two Higgs doublet models for the LHC Higgs boson data at $\sqrt{s}=7$ and 8 $\mathrm{TeV}$, arXiv: 1310.3374 [INSPIRE].

[27] A. Pich and P. Tuzon, Yukawa alignment in the two-Higgs-doublet model, Phys. Rev. D 80 (2009) 091702 [arXiv:0908.1554] [INSPIRE]. 
[28] W. Altmannshofer, S. Gori and G.D. Kribs, A minimal flavor violating 2HDM at the LHC, Phys. Rev. D 86 (2012) 115009 [arXiv:1210.2465] [InSPIRE].

[29] Y. Bai, V. Barger, L.L. Everett and G. Shaughnessy, General two Higgs doublet model (2HDM-G) and Large Hadron Collider data, Phys. Rev. D 87 (2013) 115013 [arXiv: 1210.4922] [INSPIRE].

[30] K. Cheung, J.S. Lee and P.-Y. Tseng, Higgcision in the two-Higgs doublet models, JHEP 01 (2014) 085 [arXiv: 1310.3937] [INSPIRE].

[31] A. Celis, V. Ilisie and A. Pich, LHC constraints on two-Higgs doublet models, JHEP 07 (2013) 053 [arXiv: 1302.4022] [INSPIRE].

[32] A. Celis, V. Ilisie and A. Pich, Towards a general analysis of LHC data within two-Higgs-doublet models, JHEP 12 (2013) 095 [arXiv: 1310.7941] [INSPIRE].

[33] R.A. Battye, G.D. Brawn and A. Pilaftsis, Vacuum topology of the two Higgs doublet model, JHEP 08 (2011) 020 [arXiv:1106.3482] [INSPIRE].

[34] W. Konetschny and W. Kummer, Nonconservation of total lepton number with scalar bosons, Phys. Lett. B 70 (1977) 433 [InSPIRE].

[35] T. Cheng and L.-F. Li, Neutrino masses, mixings and oscillations in $\mathrm{SU}(2) \times \mathrm{U}(1)$ models of electroweak interactions, Phys. Rev. D 22 (1980) 2860 [INSPIRE].

[36] L. Wang and X.-F. Han, $130 \mathrm{GeV} \gamma$-ray line and enhancement of $h \rightarrow \gamma \gamma$ in the Higgs triplet model plus a scalar dark matter, Phys. Rev. D 87 (2013) 015015 [arXiv:1209.0376] [INSPIRE].

[37] D. Eriksson, J. Rathsman and O. Stål, 2HDMC: two-Higgs-doublet model calculator physics and manual, Comput. Phys. Commun. 181 (2010) 189 [arXiv:0902.0851] [INSPIRE].

[38] D. Eriksson, J. Rathsman and O. Stal, 2HDMC: two-Higgs-doublet model calculator, Comput. Phys. Commun. 181 (2010) 833 [INSPIRE].

[39] Particle Data Group collaboration, J. Beringer et al., Review of particle physics, Phys. Rev. D 86 (2012) 010001 [inSPIRE].

[40] F. Mahmoudi, SuperIso v2.3: a program for calculating flavor physics observables in Supersymmetry, Comput. Phys. Commun. 180 (2009) 1579 [arXiv:0808.3144] [InSPIRE].

[41] Heavy Flavor Averaging Group collaboration, Y. Amhis et al., Averages of B-Hadron, C-Hadron and $\tau$-lepton properties as of early 2012, arXiv:1207.1158 [INSPIRE].

[42] LHCb collaboration, First evidence for the decay $B_{s}^{0} \rightarrow \mu^{+} \mu^{-}$, Phys. Rev. Lett. 110 (2013) 021801 [arXiv: 1211.2674] [INSPIRE].

[43] http://www.slac.stanford.edu/xorg/hfag/rare/2013/radll/index.html

[44] C. Geng and J.N. Ng, Charged Higgs effect in $B_{d}^{0}-\bar{B}_{d}^{0}$ mixing, $K \rightarrow \pi$ neutrino anti-neutrino decay and rare decays of B mesons, Phys. Rev. D 38 (1988) 2857 [Erratum ibid. D 41 (1990) 1715] [INSPIRE].

[45] P. Bechtle, O. Brein, S. Heinemeyer, G. Weiglein and K.E. Williams, HiggsBounds: confronting arbitrary Higgs sectors with exclusion bounds from LEP and the Tevatron, Comput. Phys. Commun. 181 (2010) 138 [arXiv:0811.4169] [INSPIRE].

[46] P. Bechtle et al., HiggsBounds-4: improved tests of extended Higgs sectors against exclusion bounds from LEP, the Tevatron and the LHC, Eur. Phys. J. C 74 (2014) 2693 [arXiv:1311.0055] [INSPIRE]. 
[47] P. Bechtle, S. Heinemeyer, O. Stål, T. Stefaniak and G. Weiglein, HiggsSignals: confronting arbitrary higgs sectors with measurements at the Tevatron and the LHC,

Eur. Phys. J. C 74 (2014) 2711 [arXiv:1305.1933] [InSPIRE].

[48] P. Bechtle, S. Heinemeyer, O. Stål, T. Stefaniak and G. Weiglein, HiggsSignals-1.1 release note, see http://higgsbounds.hepforge.org/HS-1.1_releasenote.pdf.

[49] ATLAS collaboration, Measurements of the properties of the Higgs-like boson in the $W W^{(*)} \rightarrow \ell \nu \ell \nu$ decay channel with the ATLAS detector using $25 \mathrm{fb}^{-1}$ of proton-proton collision data, ATLAS-CONF-2013-030 (2013).

[50] ATLAS collaboration, Measurements of Higgs boson production and couplings in diboson final states with the ATLAS detector at the LHC, Phys. Lett. B 726 (2013) 88 [arXiv: 1307.1427] [INSPIRE].

[51] ATLAS collaboration, Measurements of the properties of the Higgs-like boson in the four lepton decay channel with the ATLAS detector using $25 \mathrm{fb}^{-1}$ of proton-proton collision data, ATLAS-CONF-2013-013 (2013).

[52] ATLAS collaboration, Observation of an excess of events in the search for the standard model Higgs boson in the gamma-gamma channel with the ATLAS detector, ATLAS-CONF-2012-091 (2012).

[53] ATLAS collaboration, Measurements of the properties of the Higgs-like boson in the two photon decay channel with the ATLAS detector using $25 \mathrm{fb}^{-1}$ of proton-proton collision data, ATLAS-CONF-2013-012 (2013).

[54] ATLAS collaboration, Combined coupling measurements of the Higgs-like boson with the ATLAS detector using up to $25 \mathrm{fb}^{-1}$ of proton-proton collision data, ATLAS-CONF-2013-034 (2013).

[55] ATLAS collaboration, Search for the standard model Higgs boson in $H \rightarrow \tau \tau$ decays in proton-proton collisions with the ATLAS detector, ATLAS-CONF-2012-160 (2012).

[56] ATLAS collaboration, Search for the bb decay of the standard model Higgs boson in associated W/ZH production with the ATLAS detector, ATLAS-CONF-2013-079 (2013) [ATLAS-COM-CONF-2013-080].

[57] ATLAS collaboration, Search for associated production of the Higgs boson in the $W H \rightarrow W W W^{*} \rightarrow l \nu l \nu l \nu$ and $Z H \rightarrow Z W W^{*} \rightarrow l l l \nu l \nu$ channels with the ATLAS detector at the LHC, ATLAS-CONF-2013-075 (2013) [ATLAS-COM-CONF-2013-069].

[58] CMS collaboration, Evidence for a particle decaying to $W^{+} W^{-}$in the fully leptonic final state in a standard model Higgs boson search in pp collisions at the LHC,

CMS-PAS-HIG-13-003 (2013).

[59] CMS collaboration, Update of the search for the standard model Higgs boson decaying into $W W$ in the vector boson fusion production channel, CMS-PAS-HIG-13-022 (2013).

[60] CMS collaboration, $V H$ with $H \rightarrow W W \rightarrow \ell \nu \ell \nu$ and $V \rightarrow j j$, CMS-PAS-HIG-13-017 (2013).

[61] CMS collaboration, Search for SM Higgs in $W H \rightarrow W W W \rightarrow 3 l 3 \nu$, CMS-PAS-HIG-13-009 (2013).

[62] CMS collaboration, Properties of the Higgs-like boson in the decay $H \rightarrow Z Z \rightarrow 4 l$ in $p p$ collisions at $\sqrt{s}=7$ and 8 TeV, CMS-PAS-HIG-13-002 (2013). 
[63] CMS collaboration, Evidence for a new state decaying into two photons in the search for the standard model Higgs boson in pp collisions, CMS-PAS-HIG-12-015 (2012).

[64] CMS collaboration, Updated measurements of the Higgs boson at 125 GeV in the two photon decay channel, CMS-PAS-HIG-13-001 (2013).

[65] CMS collaboration, Search for the standard model Higgs boson in the dimuon decay channel in pp collisions at $\sqrt{s}=7$ and 8 TeV, CMS-PAS-HIG-13-007 (2013).

[66] CMS collaboration, Search for the standard-model Higgs boson decaying to $\tau$ pairs in proton-proton collisions at $\sqrt{s}=7$ and 8 TeV, CMS-PAS-HIG-13-004 (2013).

[67] CMS collaboration, 1546801, CMS-PAS-HIG-13-012 (1546801).

[68] CMS collaboration, Search for the standard model Higgs boson produced in association with top quarks in multilepton final states, CMS-PAS-HIG-13-020 (2013).

[69] CMS collaboration, Search for Higgs boson production in association with a top-quark pair and decaying to bottom quarks or $\tau$ leptons, CMS-PAS-HIG-13-019 (2013).

[70] CMS collaboration, Search for ttH production in events where $H$ decays to photons at 8 TeV collisions, CMS-PAS-HIG-13-015 (2013).

[71] CDF collaboration, T. Aaltonen et al., Combination of searches for the Higgs boson using the full CDF data set, Phys. Rev. D 88 (2013) 052013 [arXiv:1301.6668] [INSPIRE].

[72] D0 collaboration, V.M. Abazov et al., Combined search for the Higgs boson with the D0 experiment, Phys. Rev. D 88 (2013) 052011 [arXiv: 1303.0823] [INSPIRE].

[73] E. Cervero and J.-M. Gerard, Minimal violation of flavour and custodial symmetries in a vectophobic two-Higgs-doublet-model, Phys. Lett. B 712 (2012) 255 [arXiv:1202.1973] [INSPIRE].

[74] J. Cao, Y. He, P. Wu, M. Zhang and J. Zhu, Higgs phenomenology in the minimal dilaton model after Run I of the LHC, JHEP 01 (2014) 150 [arXiv:1311.6661] [INSPIRE].

[75] L. Wang, J.M. Yang and J. Zhu, Dark matter in little Higgs model under current experimental constraints from LHC, Planck and Xenon, Phys. Rev. D 88 (2013) 075018 [arXiv: 1307.7780] [INSPIRE].

[76] X.-F. Han, L. Wang, J.M. Yang and J. Zhu, Little Higgs theory confronted with the LHC Higgs data, Phys. Rev. D 87 (2013) 055004 [arXiv: 1301.0090] [INSPIRE].

[77] J. Reuter and M. Tonini, Can the 125 GeV Higgs be the Little Higgs?, JHEP 02 (2013) 077.

[78] J. Cao, Z. Heng, J.M. Yang and J. Zhu, Status of low energy SUSY models confronted with the LHC 125 GeV Higgs data, JHEP 10 (2012) 079 [arXiv:1207.3698] [InSPIRE].

[79] J.-J. Cao, Z.-X. Heng, J.M. Yang, Y.-M. Zhang and J.-Y. Zhu, A SM-like Higgs near 125 GeV in low energy SUSY: a comparative study for MSSM and NMSSM, JHEP 03 (2012) 086 [arXiv: 1202.5821] [INSPIRE].

[80] J.F. Gunion, Y. Jiang and S. Kraml, The constrained NMSSM and Higgs near 125 GeV, Phys. Lett. B 710 (2012) 454 [arXiv:1201.0982] [INSPIRE]. 